\title{
Enhancing power factor of SnSe sheet with grain boundary by doping germanium or silicon
}

\author{
Jie Sun ${ }^{1}$, Jiabing $\mathrm{Yu}^{2}$, Yaguang Guo ${ }^{1}$ and Qian Wang $\mathbb{1}^{1 凶}$
}

Grain boundaries (GBs) widely exist in two-dimensional (2D) and three-dimensional (3D) materials in experiment, which significantly affect the thermoelectric performance because of the scattering effect on the transport of both electron and phonon. Motivated by the research progress in 3D SnSe, we have systematically studied the GBs in a SnSe monolayer including their stable geometric configurations, the effect of GBs on power factor and Seebeck coefficient, and the strategies to improve the performance by using first principles calculations combined with semiclassical Boltzmann theory. We find that the GBs increase the potential energy barrier of carriers and decrease the valley degeneracy of the conducting bands, leading to the reduction of Seebeck coefficient, as compared to that of the pristine SnSe sheet. We further demonstrate that the trapping gap states are effectively eliminated or reduced by doping germanium or silicon, leading to the enhanced electrical conductivity, power factor, and Seebeck coefficient. These findings shed lights on developing practical strategies for modulating the thermoelectric performance of 2D polycrystalline sheets.

npj Computational Materials (2020)6:99; https://doi.org/10.1038/s41524-020-00368-6

\section{INTRODUCTION}

Thermoelectric materials have attracted worldwide attention in the last few decades because they can directly harvest electricity from waste heat. Among them, tin selenide ( $\mathrm{SnSe}$ ) single crystal is most well-known for its astonishing high ZT values of $2.6 \pm 0.3$ at $923 \mathrm{~K}$ and $2.8 \pm 0.5$ at $773 \mathrm{~K}^{1,2}$. However, the high cost of synthesizing SnSe single crystal and its poor mechanical properties impede the practical applications of this material. Therefore, many studies have been carried out for studying the thermoelectric performance of $3 \mathrm{D}$ polycrystalline $\mathrm{SnSe}^{3-9}$ and $2 \mathrm{D} \mathrm{SnSe}{ }^{10}$. It has been found that the ZT value of polycrystalline SnSe is dramatically reduced as compared to that of single-crystal SnSe because of the existence of grain boundaries (GBs) in polycrystalline structures, which can reduce the electrical conductivity. For example, GBs decrease the number of carriers in 3D p-type polycrystalline SnSe, leading to the poor electrical conductivity and poor thermoelectric performance ${ }^{11}$. Also, in n-type 3D polycrystalline $\mathrm{SnSe}$, the carrier concentration is reduced as compared with that of single crystalline $\mathrm{SnSe}^{7}$. To improve the performance, chemical doping has been used. Previous studies have shown that alkali elements ${ }^{3-5}$, halogens ${ }^{6,7}$, and $\mathrm{Ag}^{8,9}$ are the promising dopants for increasing the $\mathrm{ZT}$ value of $3 \mathrm{D}$ polycrystalline SnSe. For instance, the ZT value of K-doped polycrystalline $\mathrm{SnSe}$ is 1.1 at $773 \mathrm{~K}^{5}$, which is more than twice the original value of pure polycrystalline $\mathrm{SnSe}(0.5)$ at $823 \mathrm{~K}^{12}$, but still much less than that (2.6-2.8) of its single crystal counterpart.

For SnSe thin films and nanosheets, the $\mathrm{ZT}$ values have been experimentally found to be relatively low, the maximum value can only reach 0.28 at $675 \mathrm{~K}^{13,14}$, and the underlying reasons remain unclear. As a matter of fact, GBs have been found to exist in many $2 \mathrm{D}$ materials ${ }^{15-18}$. However, the study on the effect of GBs on the thermoelectric properties of 2D SnSe has not been reported, although significant research progress has been made in $3 \mathrm{D} \mathrm{SnSe}$ with GBs. To explore the mechanism of low ZT value of 2D SnSe, it is essential to address the following fundamental questions: (1) what are the stable GB configurations in 2D SnSe? (2) How does GB affect the thermoelectric performance of 2D SnSe? (3) What are the strategies for improving the thermoelectric performance? To this end, we carry out a systematic theoretical study. Our results show that the GBs in 2D SnSe result in the defect trapping gap states inside the band gap, which impede the mobility of carriers. Doping with $\mathrm{Ge}$ or $\mathrm{Si}$ atoms can eliminate or reduce the trapping gap states, leading to a narrow band gap, an enhanced band convergence, and an improved electrical conductivity. These features, together with the reduced thermal conductivity, endow the 2D SnSe sheet containing GBs with improved thermoelectric performance.

\section{RESULTS AND DISCUSSION}

Geometric structures

Unlike other 2D materials with well-characterized GB structures such as in graphene ${ }^{15}$, boron nitride ${ }^{16}$, phosphorene ${ }^{17}$, and $\mathrm{MoS}_{2}{ }^{18}$, the GB configurations in SnSe monolayer are still unclear. We first need to determine the stable GB configurations in the 2D $\mathrm{SnSe}$. To this end, we use the coincident-site-lattice (CSL) theory ${ }^{19}$ that has been widely applied to determine the GB configurations in many materials ${ }^{20-22}$, and find three stable $\mathrm{GB}$ configurations with the grain orientation angles of $37.76^{\circ}, 54.32^{\circ}$, and $91.48^{\circ}$, as shown in Fig. 1, where the [010] and [001] directions are chosen to be the GB edge and rotation axis, respectively. The density of GB is determined by the distance between two neighboring GBs, the larger distance leads to the lower GB density. Taking monolayer SnSe with $91.48^{\circ} \mathrm{GB}$ as an example, we choose structures with different distances between two neighboring GBs to calculate their GB energies. The result is shown in Supplementary Fig. 1. When the distance between the two neighboring GBs reaches $21.49 \AA$, the $\mathrm{GB}$ energy converges to $0.21 \mathrm{eV}$, which means when the set distances are larger than $21.49 \AA$, the GB density will not be a main factor affecting the properties. To avoid the coupling

${ }^{1}$ Center for Applied Physics and Technology, Department of Materials Science and Engineering, HEDPS, College of Engineering, Peking University, Beijing 100871, China. ${ }^{2}$ College

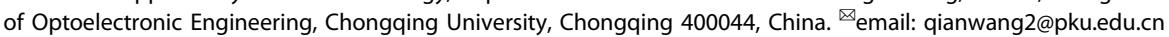



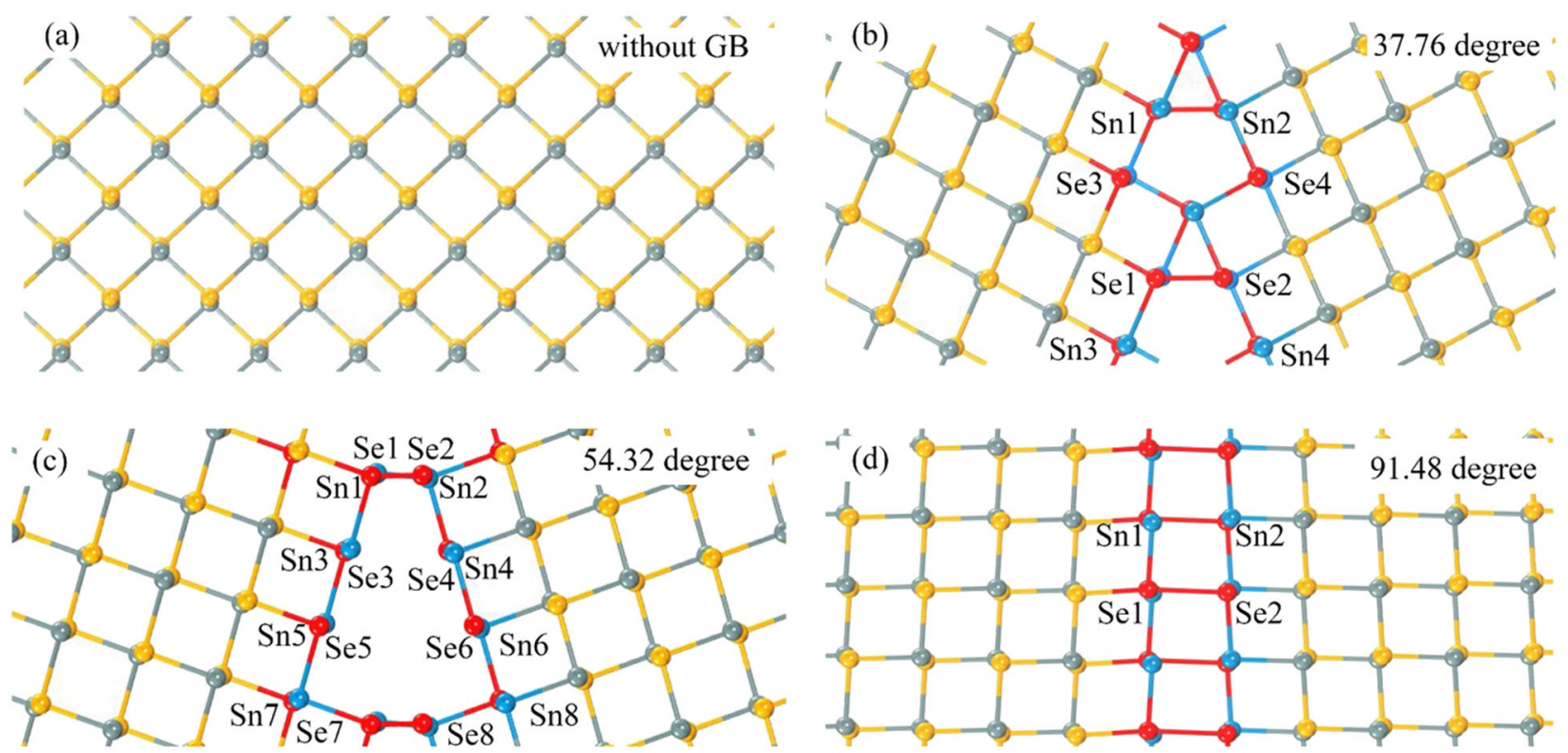

Fig. 1 Geometric structures of 2D SnSe. Top view of the optimized 2D SnSe sheet a without GB, with b $37.76^{\circ} \mathrm{GB}, \mathbf{c} 54.32^{\circ} \mathrm{GB}$, and d $91.48^{\circ}$ GB. Red and blue, cyan and yellow spheres represent the Sn and Se atoms in the GBs and grains, respectively.

between two neighboring GBs, we set the distance as $60 \AA$ in calculations. Supplementary Fig. $1 \mathrm{~b}$ is for the number of atoms versus the distances between two neighboring GBs. Also, to make DFT method handleable for our system, the 2D SnSe with $91.48^{\circ}$ $\mathrm{GB}$ is chosen for doing the calculations containing $80 \mathrm{Sn}$ and $80 \mathrm{Se}$ atoms with the lattice parameters of $a=122.78, b=6.14 \AA$. The structures with $37.76^{\circ} / 54.32^{\circ} \mathrm{GB}$ contain $84 / 62 \mathrm{Sn}$ and $84 / 62 \mathrm{Se}$ atoms with the lattice parameters of $a=58.26 / 62.21, b=13.59$ / $9.63 \AA$, respectively. The vacuum space of $18 \AA$ is included along the $c$ directions for these structures. The distances between two neighboring $\mathrm{GBs}$ are large enough to avoid their mutual interactions ${ }^{17}$. Interestingly, the GB structure with grain orientation angle of $91.48^{\circ}$ is similar to the antiphase GB in polycrystalline bulk SnSe discovered by Zhao et al. in the experiment, which is a stable GB type ${ }^{4}$, implying that it is likely to observe such GB type also in $2 D$ SnSe. We calculate the $G B$ energies of the three $G B$ structures using the formula: $E_{\mathrm{GB}}=\left(E_{1}-E_{0}\right) / 2 l$, where $E_{1}$ and $E_{0}$ are the energies of the structures with and without $G B s, l$ is the length of $\mathrm{GB}$. The $\mathrm{GB}$ energies of the $2 \mathrm{D}$ SnSe with $37.76^{\circ}, 54.32^{\circ}$, and $91.48^{\circ} \mathrm{GB}$ are $0.18,0.34$, and $0.21 \mathrm{eV} / \AA$, respectively. Because the structure with $91.48^{\circ} \mathrm{GB}$ has a similar GB configuration to that of the antiphase GBs in the synthesized polycrystalline $\mathrm{SnSe}^{4}$, and the other two GBs are also low in energy, we choose the three configurations for further calculations and discussions.

GBs usually contain some wrong arranged atoms or bonds, as shown in Fig. 1. For the structure with $37.76^{\circ} \mathrm{GB}$, the wrong arranged atoms in the GB region are labeled as Sn1-Sn2/Se1-Se2, Sn3-Sn4/Se3-Se4, and for the structure with $54.32^{\circ} \mathrm{GB}$, the wrong arranged atoms are labeled as $\mathrm{Sn} 1-\mathrm{Sn} 2 / \mathrm{Se} 1-\mathrm{Se} 2, \mathrm{Sn} 3-\mathrm{Sn} 4 /$ Se3-Se4, Sn5-Sn6/Se5-Se6, and Sn7-Sn8/Se7-Se8. The situation with wrong arranged atoms in the $91.48^{\circ} \mathrm{GB}$ is less complicated, shown as Sn1-Sn2 and Se1-Se2.

It was demonstrated that the disordered atoms or some wrong bonds in GBs can result in trapping gap states ${ }^{23}$ that are able to trap the carriers and decrease the mobility of free carriers, leading to the reduction of electrical conductivity. For instance, for an ntype semiconductor, these trapping states can be filled by electrons, and when they are accumulated, a negative charge region can be developed in the GB. The adjacent grains of the GB become positively charged and a potential energy barrier is created, thus reducing the mobility of electrons ${ }^{24}$. While, for those shallow gap states which are coupled with conducting bands, the potential energy barrier between GB and grain region is low and the detrimental effect can be reduced. To see how GB affects the electronic properties of $2 \mathrm{D} \mathrm{SnSe}$, we calculate the band structures of the 2D SnSe with the three GB types, respectively. To avoid the effect caused by the anisotropy of $2 \mathrm{D}$ SnSe, the structure without GB for comparison is also tilted by the corresponding degree from the original structure, namely, by $91.48^{\circ}$, as plotted in Supplementary Fig. 2.

\section{Electronic structures}

The band structures of the SnSe sheet with and without the $91.48^{\circ}$ GB are shown in Fig. 2a, b. To compare the changes of conduction and valence bands, we plot the band structures using band alignment scheme, where the vacuum energy level is set as zero energy. The electrostatic potentials are calculated to be 2.74, 2.73, 2.69, and $2.72 \mathrm{eV}$ for SnSe monolayer without GB, with $91.48^{\circ} \mathrm{GB}$, doped with $\mathrm{Ge} / \mathrm{Si}$, respectively, as shown in Supplementary Fig. 3. One can see that the GB results in four trapping gap states inside the band gap, which can trap the carriers, leading to the decrease of electrical conductivity. We also calculate the band structures of the $2 \mathrm{D}$ SnSe sheets with the $37.76^{\circ}$ and $54.32^{\circ} \mathrm{GB}$, respectively, as shown in Fig. 3a, d. It is interesting to see the emerging gap states are coupled with the conducting bands for the structure with $54.32^{\circ} \mathrm{GB}$. Thus, the carrier mobility is less affected. While, the defect gap states are observed in the structure with the $37.76^{\circ} \mathrm{GB}$.

To explore the origins of these gap states, the site-projected DOS of the $2 \mathrm{D}$ sheet with $91.48^{\circ}$ and $37.76^{\circ} \mathrm{GB}$ configurations are calculated, respectively. The results are plotted in Supplementary Figs. 4 and 5. For the structure with $91.48^{\circ} \mathrm{GB}$, we find four defect gap states arising from the $5 p_{z}$ orbitals of $\mathrm{Sn}$ atoms in the GB. Two wrong bonds of $\mathrm{Sn}-\mathrm{Sn}$ at the middle and another two at the edge are responsible for the four trapping gap states inside the band gap (see Supplementary Fig. 2b). For the structure with $37.76^{\circ} \mathrm{GB}$, the defect trapping gap states also originate from the $5 p_{z}$ orbitals of the $\mathrm{Sn}$ atoms in the GB. Besides the site-projected DOS, the fatband analysis is also carried out to study the origins of these emerging gap states inside the band gap. Based on the distance between the GB and Sn, four Sn atoms are chosen for doing the fat-band analysis, as shown in Supplementary Fig. 6. We note when the distance from the GB becomes larger, the contribution 

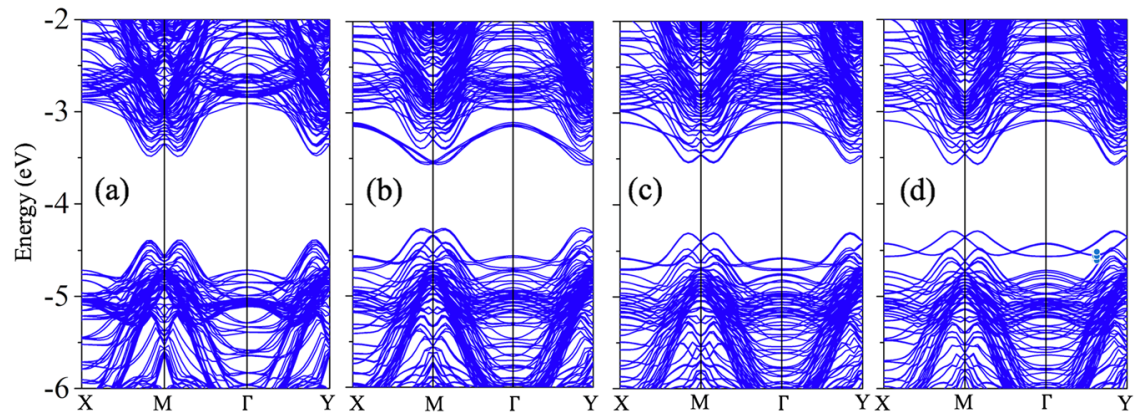

Fig. 2 Band structures of 2D SnSe with $91.48^{\circ} \mathrm{GB}$. Band structure of the 2D SnSe sheet a without $\mathrm{GB}$ and $\mathbf{b}$ with $91.48^{\circ} \mathrm{GB}$, band structure of the c, d Ge-/Si-doped 2D SnSe sheet with $91.48^{\circ} \mathrm{GB}$.
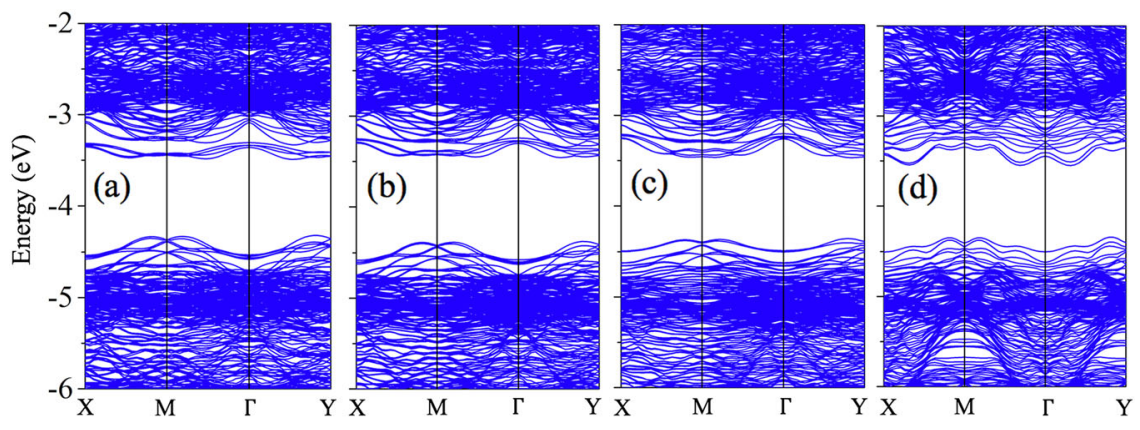

Fig. 3 Band structures of 2D SnSe with $37.76^{\circ} / 54.32^{\circ}$ GB. Band structure of a the SnSe sheet with $37.76^{\circ} \mathrm{GB}, \mathbf{b}, \mathbf{c}$ the Ge-/Si-doped SnSe sheet with $37.76^{\circ} \mathrm{GB}$, and $\mathbf{d}$ the sheet with $54.32^{\circ} \mathrm{GB}$.

to the gap states becomes smaller. Therefore, the emerging gap states are mainly attributed by the $\mathrm{Sn}$ atoms around the GB. Supplementary Fig. 7 shows the fat bands when Sn atoms at the $\mathrm{GB}$ are replaced by $\mathrm{Ge} / \mathrm{Si}$ atoms. One can see that the gap states are pushed upwards into the conduction band after doping. Since the trapping gap states are detrimental to electronic transport, a direct strategy to improve electrical conductivity is to eliminate or reduce the trapping gap states. However, less attention was paid on this point in previous studies.

Here, we choose the two GB configurations with $91.48^{\circ}$ and $37.76^{\circ}$ to study the induced gap states. According to the experiment by Sushmita et al. ${ }^{25}$, we study the effect of doping $\mathrm{Ge}$ in the GB region on the gap states. We first compare the substitution energies of $\mathrm{Ge}_{\mathrm{Sn}}$ (Ge at $\mathrm{Sn}$ site) and $\mathrm{Ge}_{\mathrm{Se}}$ ( $\mathrm{Ge}$ at Se site), which are found to -0.32 and $+1.10 \mathrm{eV}$, respectively, indicating that Ge prefers to the Sn site. So, the wrong bonds of $\mathrm{Sn}-\mathrm{Sn}$ become $\mathrm{Ge}-\mathrm{Ge}$ after the doping. We then examine the $\mathrm{Ge}$ segregation energy profile across the GB regions for the two configurations. The results show that the Ge-doping leads to a spontaneous Ge segregation in the GBs, irrespective of the GB types (see Supplementary Fig. 8a). When doped with $\mathrm{Ge}$, the $\mathrm{Sn}-\mathrm{Sn}$ wrong bonds may become Ge-Ge bonds or $\mathrm{Sn}-\mathrm{Ge}$ bonds. To find the energetic preference, we calculate their bond energies, which are found to be -0.55 and $-0.31 \mathrm{eV}$ for the Ge-Ge and $\mathrm{Sn}-\mathrm{Ge}$ bonds, respectively, suggesting that forming Ge-Ge bond is energetically more favorable over $\mathrm{Sn}-\mathrm{Ge}$ bond. The band structure of the Ge-doped sheet with $91.48^{\circ} \mathrm{GB}$ is plotted in Fig. 2c. One can see that the four trapping gap states are eliminated after the doping. The underlying reason is that the coupling of the $5 p_{z}$ orbitals of $\mathrm{Sn}$ in the $\mathrm{GB}$ region is changed to the stronger coupling of $4 p_{z}$ orbitals of Ge atoms, which can move the gap states upward to the conduction bands. Thus, the trapping gap states are now eliminated by Ge doping and the potential energy barrier cannot form across the GB. According to the calculated results, the band gap for $91.48^{\circ} \mathrm{GB}$ with Ge doping is decreased by $0.16 \mathrm{eV}$, as compared with gap in the pristine situation, due to the fact that Ge is more electronegative than $\mathrm{Sn}$. We conclude that Ge doping in the GB can eliminate the trapping gap states, resulting in the increased electrical conductivity.

It is natural to consider whether silicon ( $\mathrm{Si}$ ) can also have the similar effect because $\mathrm{Si}$ is isoelectronic to $\mathrm{Ge}$ and $\mathrm{Sn}$. In addition, $\mathrm{Si}$ is much cheaper than Ge. Interestingly, it was found that the power factor and Seebeck coefficient are enhanced when $\mathrm{Si}$ is introduced into $3 \mathrm{D} \mathrm{SnSe}{ }^{14}$. Therefore, it is possible to dope SnSe with $\mathrm{Si}$ in the experiment. We then dope $\mathrm{Si}$ in the 2D SnSe sheet with $91.48^{\circ} \mathrm{GB}$. The calculated band structure is plotted in Fig. $2 \mathrm{~d}$, which clearly shows that the trapping gap states are eliminated. The corresponding value of band gap reduction is $0.21 \mathrm{eV}$ for the structure with $91.48^{\circ} \mathrm{GB}$ after Si doping. For the structure with $37.76^{\circ} \mathrm{GB}$, we also study the effect of doping on the gap states by replacing $\mathrm{Sn}$ with $\mathrm{Ge}$ and $\mathrm{Si}$, respectively, in the $\mathrm{GB}$ region. The formation energies of $\mathrm{Ge}$ and the $\mathrm{Si}$ at $\mathrm{Sn}$ site are shown in Supplementary Fig. 8b. Again, both $\mathrm{Ge}$ and $\mathrm{Si}$ are found to have the tendency to occupy the Sn sites in the GB. The band structures are calculated and plotted in Fig. 3b, c, where the trapping gap states turn into shallow gap states coupling with the conducting bands, which are also attributed by the stronger coupling of the $4 p_{z} / 3 p_{z}$ orbitals of $\mathrm{Ge} / \mathrm{Si}$ atoms, as compared with the case of $5 p_{z}$ orbitals of $\mathrm{Sn}$ atoms. Therefore, doping $\mathrm{Ge} / \mathrm{Si}$ in the 2D SnSe with GBs can be a useful strategy to eliminate or reduce the trapping gap states, thus leading to the enhancement of electrical conductivity.

According to the above calculations, one can see that different $\mathrm{GB}$ configurations induce the different trapping gap states. From the site-projected DOS of the structures with $37.76^{\circ} \mathrm{GB}$ and $91.48^{\circ}$ $G B$, we find that the contributions to the total DOS of Sn atoms are related to their distances from the middle of GB. For instance, in Fig. $1 \mathrm{~b}, \mathrm{Sn} 1-\mathrm{Sn} 2$ contributes more than Sn3-Sn4 to the total DOS. This is understandable because their mutual coupling becomes weaker when the distance between them increases. As shown in Fig. $1 \mathrm{~b}, \mathrm{~d}$, for the structures with $37.76^{\circ} \mathrm{GB}$ and $91.48^{\circ}$ $\mathrm{GB}$, the percentages of wrong arranged atoms with small mutual 

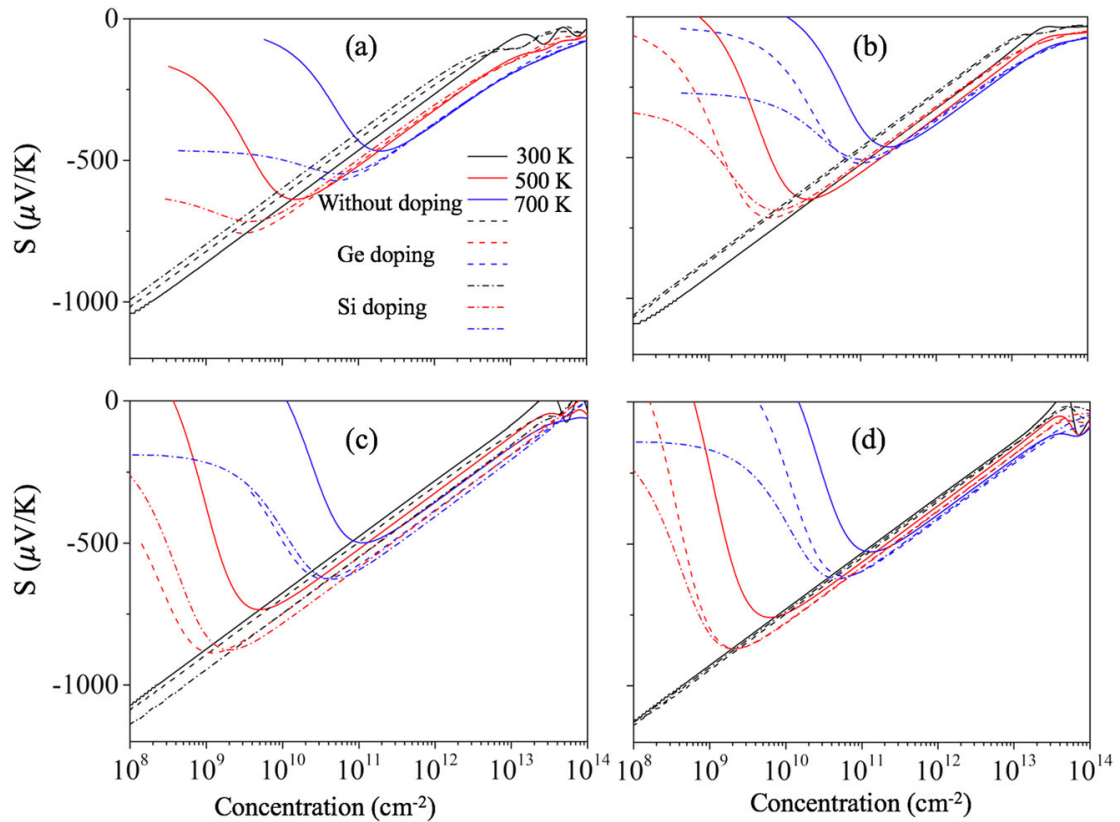

Fig. 4 Seebeck coefficients of $\mathbf{n}$-type 2D SnSe with $91.48^{\circ} \mathrm{GB}$. Concentration dependence of the Seebeck coefficients perpendicular a and parallel $\mathbf{b}$ to the GB direction of the n-type 2D SnSe sheet with $91.48^{\circ} \mathrm{GB}$. Concentration dependence of the Seebeck coefficients perpendicular $\mathbf{c}$ and parallel $\mathbf{d}$ to the $\mathrm{GB}$ direction of the n-type $2 \mathrm{D}$ SnSe sheet with $37.76^{\circ} \mathrm{GB}$, at different temperatures, respectively.

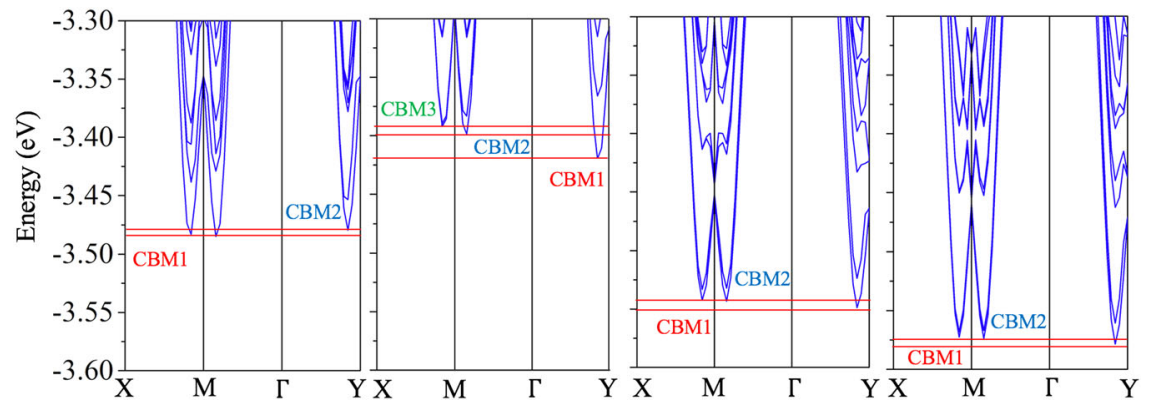

Fig. 5 Band structures of $2 \mathrm{D}$ SnSe with $91.48^{\circ} \mathrm{GB}$ in short energy range. Band structure in a short energy range for the $2 \mathrm{D}$ SnSe sheet a without $\mathrm{GB}$, b with $91.48^{\circ} \mathrm{GB}$, and for c the Ge-doped and d Si-doped SnSe sheet with $91.48^{\circ} \mathrm{GB}$.

distances (Sn1-Sn2) are 50\% and $100 \%$. While, for the structure with $54.32^{\circ} \mathrm{GB}$, the wrong arranged atoms with small distances (Sn1-Sn2 and Sn7-Sn8) occupy $40 \%$ of total atoms at GB. The density of wrong bonds with small distances may be not high enough to induce trapping gap states for the structure with $54.32^{\circ} \mathrm{GB}$.

\section{Seebeck coefficients}

The Seebeck coefficients are then calculated using Boltztrap2 code. Here, we discuss the structures with $37.76^{\circ}$ and $91.48^{\circ} \mathrm{GB}$, because in both of them trapping gap states are observed. The results for the n-type 2D SnSe are shown in Fig. 4. It is interesting to note that the Seebeck coefficients of the doped sheets increase in both parallel and perpendicular to the GB direction. According to previous studies ${ }^{26,27}$, we choose the concentration range of $10^{8}$ to $10^{14} \mathrm{~cm}^{-2}$ to calculate the Seebeck coefficients. For the structure with $91.48^{\circ} \mathrm{GB}$, the Seebeck coefficients at $500 / 700 \mathrm{~K}$ are $641.34 / 650.25,470.29 / 462.65 \mu \mathrm{V} / \mathrm{K}$ in parallel/perpendicular to the GB direction, respectively. When doped with $\mathrm{Ge}$, the corresponding values increase to 759.76/715.62, 574.28/522.92 $\mu \mathrm{V} /$ $\mathrm{K}$, as shown in Fig. 4a, b. The calculated results along the $x$ and $y$ directions are close to each other within the numerical error range. It is known that large Seebeck coefficient usually requires large DOS effective mass $\left(m_{\mathrm{d}}{ }^{*}\right)$, which is related to band effective mass $m_{\mathrm{b}}{ }^{*}$ and valley degeneracy $N_{\mathrm{v}}$ via $m_{\mathrm{d}}{ }^{*}=m_{\mathrm{b}}{ }^{*} \cdot N_{\mathrm{v}}{ }^{2 / 3}$. Therefore, Seebeck coefficient can be tuned by increasing $m_{\mathrm{b}}{ }^{*}$ or $N_{\mathrm{v}}$, while they have different effects on carrier mobility $\mu$. The relation between $m_{\mathrm{b}}{ }^{*}$ and $\mu$ is given by $\mu \propto 1 / m_{\mathrm{b}}^{* 5 / 2}$, indicating that the increase of $m_{\mathrm{b}}{ }^{*}$ is detrimental to carrier mobility and the thermoelectric performance. On the other hand, the Seebeck coefficient can be increased by increasing the valley degeneracy, without harming the carrier mobility. The valley degeneracy can be improved by the convergence of many conducting or valance bands, whose energies are close to each other. This strategy has achieved success in improving thermoelectric performance in many materials ${ }^{28-33}$. To see the conducting bands more clearly, we plot the band structures of $2 \mathrm{D}$ SnSe with and without $91.48^{\circ}$ GB in small energy ranges, as shown in Fig. 5. From Fig. 5a, for the structure without $\mathrm{GB}$, two degenerate conducting bands can be seen from $X$ tom $M$ and $M$ to $\Gamma$. The energy difference between CBM1 and CBM2 is only $0.0050 \mathrm{eV}$. When GB is induced, obvious band divergence can be noted with energy difference between CBM1 and CBM2 becoming $0.0200 \mathrm{eV}$, as seen in Fig. 5b. Also, the former two degenerate conducting bands from $X$ tom $M$ and $M$ to $\Gamma$ diverge to each other, with energy difference between CBM2 and CBM3 becoming $0.0060 \mathrm{eV}$. As shown Fig. 5c, d, when the 
trapping gap states are eliminated by doping $\mathrm{Ge}$ or $\mathrm{Si}$, the conducting bands from $X$ tom $M$ and $M$ to $\Gamma$ converge again and energy differences between CBM1 and CBM2 become 0.0057 and $0.0045 \mathrm{eV}$, respectively. The vanish of trapping states caused by doping results in the convergence of conducting bands, leading to increase of valley degeneracy. Therefore, the Seebeck coefficients can be enhanced. For the structure with $37.76^{\circ} \mathrm{GB}$, those trapping gap states become shallow gap states after doping with $\mathrm{Ge}$, coupling with original conducting bands, as shown in Fig. $3 \mathrm{~b}$. The band convergence of these shallow gap states from $X$ tom $M$ and $M$ to $\Gamma$, compared with the original conducting bands, may account for the increase in the Seebeck coefficients. We then also calculate the Seebeck coefficients of p-type SnSe sheet with and without doping. The results are shown in Supplementary Fig. 9. It is found that Ge doping can also slightly increase the Seebeck coefficients of the structures with $91.48^{\circ}$ and $37.76^{\circ} \mathrm{GB}$.

\section{Thermal conductivity}

Thermal conductivity is another important factor in determining thermoelectric performance of materials. Low thermal conductivity is needed to have a high ZT value. The thermal conductivity of monolayer SnSe was calculated to be 2.5 (2.02) W/mK along the zigzag (armchair) direction in our previous work by solving Boltzmann transport equation combined with DFT calculations ${ }^{10}$. In addition, it was experimentally found that the existence of GB and doping atoms can reduce the thermal conductivity of $2 \mathrm{D}$ $\mathrm{SnSe}^{25}$ because of the enhanced phonon scattering. So we can expect that the thermal conductivity of 2D SnSe would be reduced by introducing GBs or doping atoms, while its value remains computationally too demanding to be obtained because of the huge supercell. Therefore, the thermoelectric performance of 2D SnSe can be effectively enhanced when GB and doping are incorporated.

\section{Summary}

In summary, by studying the effect of GB on the thermoelectric performance of 2D SnSe, we find that different GB configurations can induce different defect trapping gap states inside the band gap, which can form potential energy barrier, thus impeding the mobility of carriers. Such situations can be improved by doping with $\mathrm{Ge}$ or $\mathrm{Si}$, which can effectively shift the trapping gap states upper into or close to the conducting bands due to the strong orbital coupling between two $\mathrm{Ge}$ or Si atoms, thus enhancing the electrical conductivity. Meanwhile, the band convergence in the conducting bands induced by doping can also increase the Seebeck coefficients of 2D SnSe with GBs. Furthermore, the existence of GB and the Si/Ge dopants can effectively scatter the phonons and decrease the thermal conductivity. These factors together synergistically improve thermoelectric properties of 2D SnSe through GB and doping engineering. These findings can help the design of practical thermoelectric materials that can avoid the problems of single crystal such as the high cost in synthesis and the poor mechanical properties in device processing.

\section{METHODS}

\section{Density functional theory calculations}

First-principles calculations with the framework of density-functional theory (DFT) are carried out by using the Vienna ab initio simulation package $(\mathrm{VASP})^{34,35}$, employing the projector augmented wave (PAW) pseudopotentials ${ }^{36}$. The electron exchange-correlation interaction is treated by using Perdew-Burke-Ernzerhof (PBE) functional ${ }^{37}$. The energy cutoff is set to $500 \mathrm{eV}$. The convergence criteria of total energy and final force on each atom during structural optimization are set to be 0.0001 and $0.1 \mathrm{eV} / \AA \AA$. A vacuum space of $18 \AA$ is applied to avoid the interactions between two neighboring layers in the direction perpendicular to the sheet. The Brillouin zone is sampled with $12 \times 24 \times 1$ Monkhorst-Pack kpoint meshes ${ }^{38}$.

\section{Seebeck coefficients calculations}

Electronic transport properties are calculated in the framework of the Boltzmann transport theory within the constant relaxation time approximation. The semiclassical Boltzmann theory as implemented in the Boltztrap2 code ${ }^{39}$ is used to calculate Seebeck coefficient ${ }^{40}$ defined as

$S=\frac{e}{\sigma T} \int \tau(\vec{k}) v(\vec{k}) v(\vec{k})\left(\varepsilon_{\mathrm{k}}-\varepsilon_{\mathrm{f}}\right)\left[-\frac{\partial f^{0}\left(\varepsilon_{\mathrm{k}}\right)}{\varepsilon_{\mathrm{k}}}\right] d \varepsilon$

where $\sigma$ represents the electrical conductivity, $\tau(\vec{k})$ is the relaxation time, $v(\vec{k})$ is the group velocity, $\varepsilon_{\mathrm{f}}$ is the Fermi energy and $f^{0}$ is the Fermi-Dirac distribution function. This method has been used successfully to study thermoelectric properties of other $2 \mathrm{D}$ materials ${ }^{10,26,41}$.

\section{DATA AVAILABILITY}

The datasets generated and/or analyzed during the current study are available from the corresponding author upon reasonable request.

\section{CODE AVAILABILITY}

All code used to calculate the current results is available from the corresponding author upon reasonable request.

Received: 6 January 2020; Accepted: 22 June 2020; Published online: 15 July 2020

\section{REFERENCES}

1. Zhao, L.-D. et al. Ultralow thermal conductivity and high thermoelectric figure of merit in SnSe crystals. Nature 508, 373 (2014).

2. Chang, $C$. et al. 3D charge and $2 D$ phonon transports leading to high out-of-plane ZT in n-type SnSe crystals. Science 360, 778-783 (2018).

3. Wei, T.-R. et al. Distinct impact of alkali-ion doping on electrical transport properties of thermoelectric p-type polycrystalline SnSe. J. Am. Chem. Soc. 138, 8875-8882 (2016).

4. Ge, Z.-H. et al. Boosting the thermoelectric performance of $(\mathrm{Na}, \mathrm{K})$-codoped polycrystalline SnSe by synergistic tailoring of the band structure and atomicscale defect phonon scattering. J. Am. Chem. Soc. 139, 9714-9720 (2017).

5. Chen, Y. X. et al. Understanding of the extremely low thermal conductivity in high-performance polycrystalline SnSe through potassium doping. Adv. Funct. Mater. 26, 6836-6845 (2016).

6. Zhang, Q. et al. Studies on thermoelectric properties of n-type polycrystalline SnSe1-xSx by iodine doping. Adv. Energy Mater. 5, 1500360 (2015).

7. Chang, $C$. et al. Raising thermoelectric performance of $n$-type $\mathrm{SnSe}$ via $\mathrm{Br}$ doping and $\mathrm{Pb}$ alloying. RSC Adv. 6, 98216-98220 (2016).

8. Chen, C.-L., Wang, H., Chen, Y.-Y., Day, T. \& Snyder, G. J. Thermoelectric properties of p-type polycrystalline SnSe doped with Ag. J. Mater. Chem. A 2, 11171-11176 (2014).

9. Luo, Y. et al. High thermoelectric performance in polycrystalline SnSe Via dualdoping with $\mathrm{Ag} / \mathrm{Na}$ and Nanostructuring With $\mathrm{Ag}_{8} \mathrm{SnSe}_{6}$. Adv. Energy Mater. 9, 1803072 (2019).

10. Wang, F. Q., Zhang, S., Yu, J. \& Wang, Q. Thermoelectric properties of singlelayered SnSe sheet. Nanoscale 7, 15962-15970 (2015).

11. Chen, Y.-X. et al. Understanding of the extremely low thermal conductivity in high-performance polycrystalline SnSe through potassium doping. Adv. Funct. Mater. 26, 6836-6845 (2016).

12. Sassi, S. et al. Assessment of the thermoelectric performance of polycrystalline $p$ type SnSe. Appl. Phys. Lett. 104, 212105 (2014).

13. Song, L., Zhang, J. \& Iversen, B. B. Enhanced thermoelectric properties of SnSe thin films grown by single-target magnetron sputtering. J. Mater. Chem. A 7, 17981-17986 (2019).

14. Ju, H. \& Kim, J. Effect of SiC ceramics on thermoelectric properties of SiC/SnSe composites for solid-state thermoelectric applications. Ceram. Int. 42, 9550-9556 (2016).

15. Liu, Y. \& Yakobson, B. I. Cones, pringles, and grain boundary landscapes in graphene topology. Nano Lett. 10, 2178-2183 (2010).

16. Liu, Y., Zou, X. \& Yakobson, B. I. Dislocations and grain boundaries in twodimensional boron nitride. ACS Nano 6, 7053-7058 (2012). 
17. Liu, Y., Xu, F., Zhang, Z., Penev, E. S. \& Yakobson, B. I. Two-dimensional monoelemental semiconductor with electronically inactive defects: the case of phosphorus. Nano Lett. 14, 6782-6786 (2014).

18. Sangwan, V. K. et al. Gate-tunable memristive phenomena mediated by grain boundaries in single-layer $\mathrm{MoS}_{2}$. Nat. Nanotechnol. 10, 403 (2015).

19. Ogawa, H. GBstudio: a builder software on periodic models of CSL boundaries for molecular simulation. Mater. Trans. 47, 2706-2710 (2006).

20. Xu, J., Liu, J. B., Liu, B. X., Wang, J. F. \& Huang, B. Defect engineering of grain boundaries in lead-free halide double Perovskites for better optoelectronic performance. Adv. Funct. Mater. 29, 11 (2019).

21. Yan, Y. et al. Electrically benign behavior of grain boundaries in polycrystalline CulnSe $e_{2}$ films. Phys. Rev. Lett. 99, 4 (2007).

22. Yin, W. J. et al. Engineering grain boundaries in $\mathrm{Cu}_{2} \mathrm{ZnSnSe}_{4}$ for better cell performance: a first-principle study. Adv. Energy Mater. 4, 7 (2014).

23. Seto, J. Y. The electrical properties of polycrystalline silicon films. J. Appl. Phys. 46, 5247-5254 (1975).

24. Bachmann, M., Czerner, M. \& Heiliger, C. Ineffectiveness of energy filtering at grain boundaries for thermoelectric materials. Phys. Rev. B 86, 115320 (2012)

25. Chandra, S. \& Biswas, K. Realization of high thermoelectric figure of merit in solution synthesized 2D SnSe nanoplates via Ge alloying. J. Am. Chem. Soc. 141, 6141-6145 (2019).

26. Sharma, S., Singh, N. \& Schwingenschlogl, U. Two-dimensional tellurene as excellent thermoelectric material. ACS Appl. Energ. Mater. 1, 1950-1954 (2018).

27. Li, T. W., Yu, J. B., Nie, G., Zhang, B. P. \& Sun, Q. The ultralow thermal conductivity and ultrahigh thermoelectric performance of fluorinated $\mathrm{Sn} 2 \mathrm{Bi}$ sheet in room temperature. Nano Energy 67, 8 (2020).

28. Pei, Y. et al. Convergence of electronic bands for high performance bulk thermoelectrics. Nature 473, 66 (2011).

29. Liu, W. et al. Convergence of conduction bands as a means of enhancing thermoelectric performance of n-type Mg2Si1- xSnx solid solutions. Phys. Rev. Lett. 108, 166601 (2012).

30. Tan, G. et al. Valence band modification and high thermoelectric performance in SnTe heavily alloyed with MnTe. J. Am. Chem. Soc. 137, 11507-11516 (2015).

31. Tang, Y. et al. Convergence of multi-valley bands as the electronic origin of high thermoelectric performance in CoSb 3 skutterudites. Nat. Mater. 14, 1223 (2015).

32. Tang, J. et al. Maximization of transporting bands for high-performance SnTe alloy thermoelectrics. Mater. Today Phys. 9, 6 (2019).

33. Yao, Z. et al. Solute manipulation enabled band and defect engineering for thermoelectric enhancements of SnTe. InfoMat 1, 571-581 (2019).

34. Kresse, G. \& Furthmüller, J. Efficient iterative schemes for ab initio total-energy calculations using a plane-wave basis set. Phys. Rev. B 54, 11169-11186 (1996)

35. Kresse, G. \& Furthmüller, J. Efficiency of ab-initio total energy calculations for metals and semiconductors using a plane-wave basis set. Computational Mater. Sci. 6, 15-50 (1996).

36. Blöchl, P. E. Projector augmented-wave method. Phys. Rev. B 50, 17953-17979 (1994).

37. Perdew, J. P., Burke, K. \& Ernzerhof, M. Generalized gradient approximation made simple. Phys. Rev. Lett. 77, 3865-3868 (1996).

38. Monkhorst, H. J. \& Pack, J. D. Special points for brillouin-zone integrations. Phys. Rev. B 13, 5188-5192 (1976).

39. Madsen, G. K. H., Carrete, J. \& Verstraete, M. J. BoltzTraP2, a program for interpolating band structures and calculating semi-classical transport coefficients. Comput. Phys. Commun. 231, 140-145 (2018).
40. Madsen, G. K. H. \& Singh, D. J. BoltzTraP. A code for calculating band-structure dependent quantities. Comput. Phys. Commun. 175, 67-71 (2006).

41. Kim, J. Y. \& Grossman, J. C. High-efficiency thermoelectrics with functionalized graphene. Nano Lett. 15, 2830-2835 (2015).

\section{ACKNOWLEDGEMENTS}

This work is partially supported by grants from the National Science Foundation China (Grants No. NSFC-21773004 and NSFC-11974028), and the National Key Research and Development Program of the Ministry of Science and technology of china (Grant No. 2017YFA0205003), and is supported by the High-Performance Computing Platform of Peking University, China. Y.G. acknowledges the Project funded by China Postdoctoral Science Foundation (No. 2019M650289).

\section{AUTHOR CONTRIBUTIONS}

Q.W. conceived and designed the project. J.S. performed the calculations of electronic properties. J.S. and J.Y. performed the calculations of Seebeck coefficient. J.S. and Y.G. prepared the structures of SnSe with grain boundaries. All authors contributed to the revisions and discussions of the paper.

\section{COMPETING INTERESTS}

The authors declare no competing interests.

\section{ADDITIONAL INFORMATION}

Supplementary information is available for this paper at https://doi.org/10.1038/ s41524-020-00368-6.

Correspondence and requests for materials should be addressed to Q.W.

Reprints and permission information is available at http://www.nature.com/ reprints

Publisher's note Springer Nature remains neutral with regard to jurisdictional claims in published maps and institutional affiliations.

(i) Open Access This article is licensed under a Creative Commons adaptation, distribution and reproduction in any medium or format, as long as you give appropriate credit to the original author(s) and the source, provide a link to the Creative Commons license, and indicate if changes were made. The images or other third party material in this article are included in the article's Creative Commons license, unless indicated otherwise in a credit line to the material. If material is not included in the article's Creative Commons license and your intended use is not permitted by statutory regulation or exceeds the permitted use, you will need to obtain permission directly from the copyright holder. To view a copy of this license, visit http://creativecommons. org/licenses/by/4.0/.

(c) The Author(s) 2020 